\title{
Spatial transcriptomics coming of age
}

cf

genes with

spatially

patterned gene

expression as

known and

novel markers

of different

cell types
For comprehensive molecular understanding of tissues, spatial transcriptomics methods aim to characterize gene expression profiles while retaining information of the spatial tissue context. Two complementary new methodologies bring us an important step closer to the goal of achieving transcriptome-wide data at single-cell resolution.

Spatial transcriptomics methods are generally divided into two main types, each with notable strengths and limitations. In fluorescence in situ hybridization (FISH)-based methods, transcripts are directly labelled in tissue sections, meaning that their single-cell (and even subcellular) location can be visualized. However, molecular crowding within cells leads to spatial overlap of fluorescence signals when more than a few hundred transcript species are visualized simultaneously. By contrast, methods based on single-cell RNA sequencing (scRNA-seq) can profile whole transcriptomes. However, as cells are dissociated before scRNA-seq, linking the transcriptomes back with their original location at resolutions approaching the single-cell level has been challenging.

In their study, Eng et al. extended the sequential FISH (seqFISH) method, previously developed in their laboratory, in which DNA probe sets targeting transcripts of interest are labelled with fluorophores.

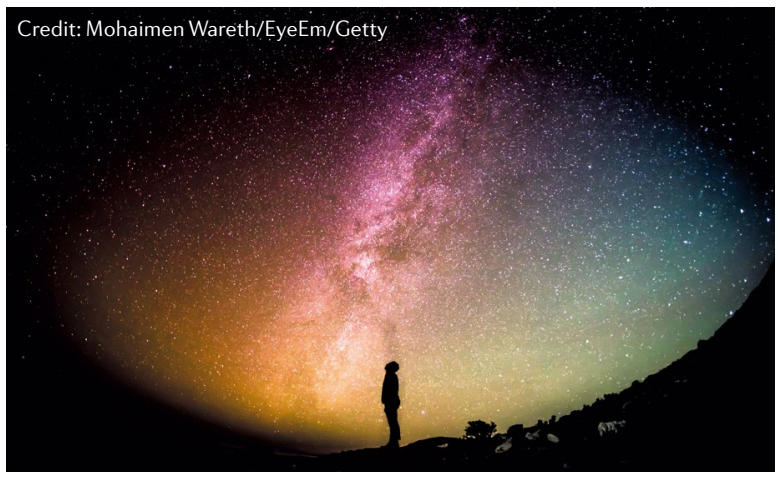

As there are only a few discernible fluorophore colours (such as the four or five used in seqFISH), serially reprobing the same slide using probe sets with known switches of probe colour allows several hundred transcripts to be distinguished based on the sequential order of colours visualized by microscopy.

In the latest work reporting seqFISH+, instead of transcriptspecific primary probes being already labelled with fluorophores, they contain four barcode sequences that serve as target sites for fluorescently labelled secondary probes. Transcripts are identified according to which secondary probes bind to these four barcode sites during four sequential rounds of probing known as 'barcoding.

Crucially, each round of barcoding is subdivided into 20 serial hybridizations. Each hybridization targets only one-twentieth of the transcript set and uses three colours of secondary probe such that only one-sixtieth of the transcripts are visualized per image. This adaptation is the key to reducing optical crowding problems, and the separate images can subsequently be merged computationally.

Eng et al. demonstrated the feasibility of seqFISH+ by designing transcript probe sets for 10,000 genes. Initially on cultured mouse cells, they showed that gene expression profiles were quantitatively equivalent to RNA-seq data. Moreover, seqFISH+ could detect expected cell layers in slices of mouse brain regions, where the team also characterized cell types based on gene expression profiles and the spatial relationships between these cell types. Finally, the authors analysed ligand-encoding and receptor-encoding RNAs in adjacent cells to identify putative intercellular ligand-receptor pairs that are dependent on local tissue context.
In their study, Rodriques, Stickels et al. enabled high-resolution spatial capture with Drop-seq beads. In the original Drop-seq method, single cells are encapsulated in droplets with a barcoded bead that labels transcripts with cell-specific barcodes but without spatial information. In the spatial adaptation, termed Slide-seq, the investigators first arrayed barcoded beads on a solid surface to make 'pucks', followed by a sequencing run (leaving the puck intact) to identify the barcodes present at each position. A tissue section is then placed on the puck, RNA is released through tissue digestion and RNA-seq libraries are produced that incorporate this position-specific barcode.

Although each bead is not strictly capturing RNA from an isolated single cell (unlike the original Drop-seq approach), $10 \mu \mathrm{m}$ beads are packed in a dense array, which is on the order of the tissue spacing of mammalian cells.

The authors demonstrated the feasibility of Slide-seq in various tissue contexts, such as by identifying known tissue structures in several mouse and human organs. Focusing on mouse brain sections, the team identified genes with spatially patterned gene expression as known and novel markers of different cell types; furthermore, within various cerebellar cell types, they found spatially defined subpopulations of cells. Finally, monitoring the time course of response in a mouse brain injury model, the authors reported that proliferative effects occurring during the initial days are followed by differentiation events over weeks.

These spatial transcriptomics methods have great potential to provide detailed molecular maps when applied across a diverse variety of tissue systems.

Darren J. Burgess

ORIGINAL ARTICLES Eng, C.-H. L. et al. Transcriptome-scale super-resolved imaging in tissues by RNA seqFISH+. Nature 568, 235-239 (2019)|Rodriques, S. G., Stickels, R. R. et al. Slideseq: a scalable technology for measuring genomewide expression at high spatial resolution. Science 363, 1463-1467 (2019) 